\title{
Big Data Applications in Power Industry
}

\section{Chao Chen ${ }^{a}$, Dongshu Yang ${ }^{b}$, Shaochen Wang ${ }^{c}$, Desheng Yang ${ }^{d}$}

\author{
Anhui Nari Jiyuan Software co., Itd of NARI group corporation, Anhui Hefei, China \\ a chen-chao6@sgepri.sgcc.com.cn, byangdongshu@ \\ sgepri.sgcc.com.cn ${ }^{c}$ wangshaocheng@sgepri.sgcc.com.cn, d yangdesheng@ sgepri.sgcc.com.cn
}

Keywords: big data, power industry, data mining.

\begin{abstract}
Power industry promotes the transformations to achieve a strong and excellent strategic objective. It proposed the system construction. Building the system is not only the upgrading and transformation of power facilities, but also a comprehensive and in-depth innovation of power operation mode and business model. Firstly, it makes information and communication technologies fuse rapidly with power production and enterprise management with unprecedented breadth and depth, at the same time it makes the services coordinate better and the business flow and information flow tend to be consistent; Power industry's data will further enrich and expand from the aspects of timeliness. Second, taking full advantage of these data based on actual grid, such as in-depth analysis of it and digging deeper worthy, can provide a lot of high value-added services. These value-added services will help power companies refine operational guidance, improve the management level and operational efficiency, and generate a lot of innovative businesses.
\end{abstract}

\section{Introduction}

With power industry's development, it built a structured data centers, unstructured data management platform, massive history/quasi-real-time data management platform, power industry geographic information service platform has accumulated a large amount of data resources, grid from the total amount and types of data are large-scale, have a good data base and initial implementation of enterprise-class data integration and sharing of resources utilization [1].

\section{Applications}

Big Data analysis application in the planning field. Planning focus is to straighten out the subject management, planning, implementation planning centralized management, in accordance with unified planning, classification management requirements, clear at all levels of the division of responsibilities, the establishment of a unified planning system covering all voltage levels and of the special, each level[2]. Strengthen the planning and programming of the organic convergence, network planning and economic and social development planning organic convergence. Big business planning capacity can be subdivided into 4 zero business ability, a 9 level of ability, comprehensive coverage of the development planning department of planning and management, plan management, comprehensive analysis, planning and design of the business. With the integration of the power industry network planning design, planning management and integrated application of information resources gradually, Historical data grid resources and related production, management of data aggregation planning large, the operating system will save run massive data, which will be the original resources of power system planning and management for analysis, decision-making and management of the most valuable [3].

At present, the amount of data integration of power system planning and management platform accumulation was GB, and the data types are mainly structured data, which contain only a small amount of text, pictures and other unstructured information. At the same time, data update frequency is not high, the real-time requirements of data is low. In analyzing the data, generates statistical reports for multi-dimensional display, and uses the power of professional models and tools for scientific analysis. After 3 years is expected, with the distribution network planning business to further expand the coverage, would gradually accumulate class TB data, for the data collection, 
storage and analysis of treatment will be put forward higher requirements. Then through the big data technology to further enhance the application value.

Electricity demand forecasting

A large amount of historical data based on the changes of regional planning area, population, history, history of national economic data, three production scales, forecast the regional electricity consumption, as further planning and design basis[4].

Spatial load forecasting

Each cell in the whole network area, land use types, based on the volume rate, industry construction area load density, covers an area of load density, small target, small target area construction area, the total load, numerical value industry load value, and the load to predict the future.

Analysis of multiple index

From a plurality of external system (such as GIS, PMS, OMS) grasping time required consistency slice data, comprehensive analysis using, so as to support the planning and design [5].

Big Data application analysis in the constructing field. Construction field focuses on optimizing the existing power grid construction organization and management mode, and establishing management system of the following: Headquarters making decision, Provincial branches acting as a project legal person, and other branches going performing. Meanwhile, implementing unified construction standard, management process and technical specification, and strengthening control on safe, quality, rate of process and cost. The infrastructure management system going through two levels of deployment started running from 2009, and staying on the internal integration stage [6]. After this stage, the system will turn to one level of deployment.

The average amount of data in infrastructure management system is about 100GB, and increases at 50GB per year. After the system turn to one level of deployment, Cumulative quantity reach TB level. The data types in the system are structured data and unstructured data. In the management of the construction of unstructured data include the document file and the scene photos, now the system has connected to structured datacenter and unstructured data platform, and the data has saved in the datacenter and platform.

In the application, the system manages about 2000-3000 projects each year, but can only a few photos because of limited storage capacity, which lead to management can't fully understand project work detail. At the same time, because the system lacks automated analysis of the application and decision support, the data can't be used effectively.

Next big data center technology in the construction application can mainly be improved in the following aspects:

Improve the system of unstructured data storage capacity, and expand the scope of the field information collection, to promote comprehensiveness and accuracy of management of construction projects

Through the technology of big data, gradually forming and improving the analysis of large amounts of unstructured data processing capacity. Based on this ability, adopting auto contrast, pattern recognition, such as mining technology, supplying automated intelligent analysis and monitoring functions to construction management process [7].

Big Data application analysis in the maintenance field. Maintenance is to build up intensive and efficient integrated production management system of operation and maintenance and based on whole life cycle of assets management business process of modern production, improve the level of equipment maintenance as the core of the lean production management.

The evaluation of equipment status, the overhaul of equipment state is the key to maintenance application, current system is mainly used in eleven power transmission and transformation equipment, system has deploy across the 20 provinces, the evaluation of system mainly semi-automatic at present, mainly adopts according to its equipment evaluation guideline for accumulate the rating status value[8].

There are many kinds of access methods of device status information. At present the most commonly used are various sensors, to obtain device status information by real time or on a regular 
basis. Video is special kinds of sensors; it can be an important and effective means that gain device status information. With the development of intelligent video analysis technology, video also can implement function that other sensor does not include.

With big data technology, we can mainly improve in the following aspects:

state evaluation: With integrated variety of basic and monitoring data, together with the amount of device status identify by intelligent recognition technology from video data, comprehensive evaluation model can be established to arrive at the overall health status of the device;

trend analysis: we research trends based on historical data analysis algorithms to build the device health status reflects the mathematical model of equipment failure trend analysis, control equipment risk;

real-time alarm: The techniques that video surveillance-based automatic inspection and safety equipment prevention can found changes in appearance, meter changes, fever defects, illegal invasion, objects near the scene pyrotechnics and other equipment health risk factors, real-time alarm, to ensure normal operation of equipment;

Maintenance, technical innovation, overhauls Decision Support: Depending on the device evaluation results, we optimizing maintenance strategy for the technological transformation, overhaul planning, screening assessment to provide basis for decision making.

Big data application analysis in operation field. Operation mainly implement all levels of Scheduling control integration which integrate national power control centre and region power system control center, improve the ability to manage large power grid and optimize the allocation of resources in a wide range of capabilities. Operation system which all named D5000 platform was integrated from multiple subsystems before, it is combined of four sub systems: energy management system, outage planning System, safe check system and outage management system.

Device state management is an important business module of power industry in operation field. It can be verified on the plan type applications and provides the perfect proposal. It provides decision support for the dispatching of power industry and enhances security and reliability of the grid. Device status management currently only can record the current device status based only on other scheduling services[9]; it has no equipment cross-section of historical inquiry and future state of the predictive analysis. With dispatch further improve operational practices, upgrading of equipment state module increasingly urgent demand.

Device status management based on power industry equipment's accounting information, equipment topology information, remote signaling device telemetry information and other relevant information. It can be the following three big data applications.

History Timetable: to achieve mass storage device data directly to a fast query time device status and device operating history;

Device data quality analysis: an integrated device information section draw current device status, device status for systems interoperability seized more than right, alert other systems or functions are checked and checked;

Predict future time sections: historical status based on mass data storage, automatic summarization dispatching rules section at any time based on changes in device status automatically collected scheme involving class information, intelligently organized, figuring out where the next period of time scheduling operations course, the final prediction device power section at a time.

Big data applications in marketing field. Marketing field focus on optimizing existing marketing organization model, expand intelligence-oriented, interactive services, accelerate the construction of information collection system, scientific allocation metering, charging and service resources to achieve metrological verification and distribution, services, and other services to the province of 95598 level focus, building marketing inspection monitoring system, the implementation of a unified business model, service standards and workflows. The power industry marketing business-related support systems are one of the core marketing SG186 engineering business systems, as well as responsible for the user-side automatic acquisition of electric energy consumption data collection system, supporting marketing data everyday statistical work such as decision support system. Which is responsible for marketing business system business expanding, tariff collection and 
other daily business accounting process control, the user is responsible for the information collection system information collection for the marketing business electricity tariff accounting system to provide data, decision support systems for the completion of statistical reporting and other functions. In addition, in order to meet the new demand marketing business, the relevant provincial companies such as the construction of intelligent customer file management system for the supply contract, the user identification information such as paper scanning achieves for easy retrieval and search. Of the core marketing system, and is responsible for the user side automatic electric energy acquisition of electricity information collection system, support marketing data daily statistical work of the auxiliary decision system, etc. Business system is responsible for the marketing industry accounting reporting for expanding, electricity seized and the day-to-day operations of process.

Data related marketing systems mainly includes: marketing organization structured data of the state of grid, electricity customers archival data, the state of grid marketing field equipment data included lines, transformers, Tai area and other, ledger data of customer service processes, metering data, tariff data, operational documents and pictures video data, and so on. With more and more number of customers, data of marketing services related systems increased dramatically. For example, with full coverage for all collected information, collection system of Zhejiang electric power industry, as of December 2012, the province's information collection data reached approximately 6TB, increased in 2TB only at 2012, in future the number of users will increase to 22 million, the amount of data will further accelerate the growth of the scale and the data volume will reach PB level[10].

With the amounts of data related marketing systems increased dramatically, it takes tremendous challenge to the data storage, analysis and processing, statistical calculations. According to marketing business, information system and its data status and needs, big data could enhance and promote the marketing with the following ways: access and efficient distributed parallel computing of marketing massive data.

For depth of excavation marketing data value from the management, customer service, business development, etc. in different directions to promote marketing business improvement, including management direction of the main cable loss analysis, electrical anomaly analysis, measurement and intelligence gathering equipment operation and maintenance; customer service orientation mainly for electricity customers with targeted electricity optimization recommendations to promote the user to save electricity; business development direction of actively planning data products to guide and promote the power industry from "sell electricity" to "also sell electricity" development.

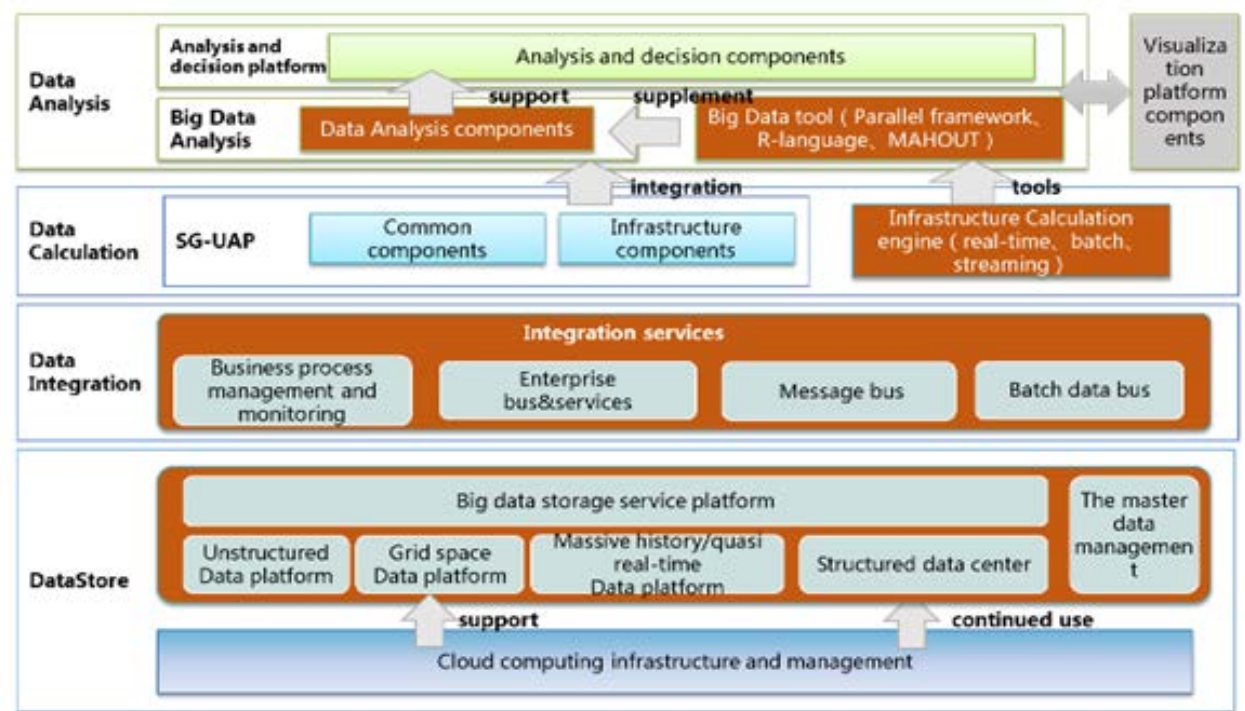

Fig.1. Big data capacity to implement the preliminary technology solutions

Application and analysis of Big Data in operation monitoring (control) center. State Grid two operations monitoring (control) center to serve as the power industry's strategic objectives to core resources and control of major business activities as the key to information technology tools to 
support and build all-weather, all-round, the whole process of integrated management of operational monitoring (control) platform. Big data capacity to implement the preliminary technology solutions is presented on Figure 1.Operation monitoring (control) system consists of five modules: Business monitoring, operational analysis, coordination and control, integrated management, large screen visualization. Currently these monitoring operations were mainly dominated profession of indicators for monitoring, does not support multi-disciplinary comprehensive monitoring indicators, in addition to transmission and transformation equipment condition monitoring and power quality monitoring outside most of the monitoring system for monitoring indicators based on a monthly, index less frequent. With the comprehensive construction of transportation supervisor, detailed data will also be access, data will show exponential growth. Operation monitoring system in terms of data transported a total of two levels, the first layer: structured data, unstructured data, and geospatial data. Second layer: structured data into metadata and coding, business data, business processes, data, supporting the functional data, the volume of data typical provincial companies will reach more than 500 GB; Unstructured data into file data, media data, the volume of data typical provincial companies will reach more than $300 \mathrm{~GB}$; geospatial data into GIS data, electricity business coordinate data, other types of data typical provincial companies will reach more than 300 GB. There are some basic business applications, missing data and data quality issues; need to further promote the context of big data big data governance, strengthening of structured and unstructured data quality management, operational monitoring data to ensure timely, complete and accurate; operation monitoring (control) center supervisor will achieve two-line monitoring, online calculation, online analysis, timeliness requirements are relatively high; various types of analytical and decision support system, the basic lines in accordance with professional conduct within the scope of the analysis of the profession, there is no established system data with other professionals the relationships, resulting in a lack of multi-disciplinary analysis and forecasting model.

Next big data center technology in the operations monitoring (control) center application can mainly be improved in the following aspects:

The context of building big data online monitoring, online analytics and online computing workstations, to meet the daily monitoring, coordination control and integrated management needs. Construction of two operational headquarters and provincial operation monitoring (control) center, to achieve the power industry's management 24 hours real time online monitoring and analysis, timely detection of power industry operations and problems in the course of the transaction and automatic warning;

Multi-disciplinary association analysis across business. In order to meet the transport monitoring across business needs multi-disciplinary analysis and mining in large data environment, the urgent need to build a variety of business models and analysis of mining algorithms, the formation of large data model libraries and algorithms library, the use of clustering and pattern recognition techniques to achieve operational monitoring multi-service association analysis, such as marketing and financial revenue and expenditure modeling for association analysis. Monitoring data and diagnostic system to monitor the quality of the object. Through the "big data" environment, the mass of heterogeneous data in real-time monitoring and analysis, in the whole process of data processing and improve data quality, continuous improvement of data availability, to expand the traditional decision analysis provide a guarantee.

Application and Analysis of Big Data in Customer Service Center. Customer Service Center is the power industry's resource optimization and integration services, to create "full-service, all-weather, service specialization, lean management, diversification," the electricity service platform. The Core business of Customer Service Center System is divided into three separate systems: 95598 intelligent interactive website, 95598 business support systems. Business supporting system of 95598 uses a nationwide deployment mode. According to the type of data to points, there are two categories, one is a file, transaction and GIS and other structured data, and about 3TB, increasing to approximately $1 \mathrm{~GB}$; there is a class voice audio class unstructured data, in document form preservation, and about nearly 10TB, annual growth 7TB, and has accelerated the trend. 
Operations management personnel during the current customer representative service quality monitoring, and take a sample survey recording papers, and select Services Typical (including pros and cons) into the knowledge base as a model case, can't be based on current and historical real-time service quality audio files effectively regulation; agent answers or rely on manual search, the answer does not implement intelligent matching and automatic push. 95598 interactive website currently only has some business functions, only the basic release announcement and does not reflect the majority of users on a timely announcement of the actual demand.

Next big data center technology in the customer service center application can mainly be improved in the following aspects:

Service quality real-time monitoring. Using big data technology to realize the quasi-real-time service calls / real-time monitoring and analysis, through the process of real-time customer service quality, to maximize protection of the quality of service calls;

Focus on hot issues. using big data techniques excavation period of time users concerned about the hot and promptly respond to and process, improve customer satisfaction;

Seating answer intelligent pushing. Manual search answers difficult to meet the requirements of timeliness, the use of big data technology to achieve intelligent answers and automatically pushed to match the seats.

\section{Conclusions}

According to the needs of the business situation, big data of analysis enhances the value of the system. Planning field designs and manages to improve the intelligent decision-making capacity; Construction field achieves the refinement, automation and intelligent monitoring management for projects; Comprehensive equipment Other state variables, the evaluation device status, to achieve state maintenance, equipment status forecast, equipment malfunction status alarm function, support to carry out overhaul business better; Achieve data quality analysis equipment running status, future devices section forecasting, optimization and validation schedule planning and implementation. Marketing data mining capabilities to enhance the value of data visualization and marketing level; enhance the audio category of unstructured data such as voice recognition capabilities and storage technology, and ultimately improve customer service quality; it can improve data quality, online monitoring, online analysis and online computing power.

\section{References}

[1] Big data the next frontier for innovation competition and productivity, Jun 9-12, 2008, Vancouver, BC. USA: ACM SIGMOD/PODS, 2008,pp.251-263.

[2] R. Buyya, C. S. Yeo, S. Venugopal. Market-oriented cloud computing: vision, hype, and reality for delivering IT service as computing utilities// Proceedings of the 10th IEEE International Conference on High Performance Computing and Communications, Sept 25-27, 2008, Dalian, China. Los Alamitos, CA, USA: IEEE CS Press, 2008.pp.11-18

[3] Gong Xueqing, Jin Cheqing, Wang Xiaoling, et al. Data-intensive science and engineering: requirements and challenges [J]. Chinese Journal of Computers, 2012, 35(8),pp.1563-1578.

[4] Chen Kang, ZhengWeimin. Cloud computing: system instances and current research [J]. Journal of Software, 2009, 20(5),pp.1337-1348.

[5] Report: Haiyan Power Supply Bureau sent a letter to thank our team to support large data. Jan 30, 2013,pp.4-8.

[6] Report: China Internet Development Report2012,pp.3-19.

[7] State Grid Corporation of the mail system user behavior data reports [J].State Grid Information \& Communication Co., Ltd. Fri, 2012,pp.35-43.

[8] Intel China Big Data Cloud Best Practices [J].Jason Dai, Yuanhao Sun, 2012,pp.234-246.

[9] Robert Fonow.In charge of big data - to grasp the pulse of the Information Age. China Brief, Dec 19, 2012,pp.135-141. 